\title{
'Selfies' Reveal Systematic Deviations from Known Principles of Photographic Composition
}

\author{
Nicola Bruno ${ }^{1}$, Valentina Gabriele ${ }^{1}$, Tiziana Tasso ${ }^{1}$ and Marco Bertamini ${ }^{2, *}$ \\ ${ }^{1}$ Dipartimento di Neuroscienze, Università di Parma, Italy \\ ${ }^{2}$ Department of Psychological Sciences, University of Liverpool, UK
}

Received 30 March 2013; accepted 4 December 2013

\begin{abstract}
We used 'selfies', self-portraits taken with a hand-held smartphone camera, to test three known principles of photographic composition: The rule of thirds, the golden ratio rule, and the eye centering principle. Although they are often taught and discussed, the origin of these principles remains unclear. It is possible that they stem from constraints on human perceptual processes. Alternatively, these principles might serve more practical purposes, such as forcing photographers to explore all quadrants of the image. Selfies provide an ideal test bed for these questions due to the control they give self-photographers when they compose the photograph. We used a database of images created by non-professional photographers $(N=388)$. After analysis, we conclude that there little support for any of the three principles, suggesting that none is strongly rooted in spontaneous perceptual preferences.
\end{abstract}

Keywords

Composition, photography, rule of thirds, golden ratio

\section{Introduction}

Since the advent of smartphones equipped with quality cameras and preview screens, a large number of individuals have all been taking pictures of themselves for purposes ranging from the social to the professional. Smartphone self-portraits, or 'selfies' as baptized in social media tags, are increasingly attracting media attention and sociological scrutiny (Fausing, 2013; Houghton et al., 2013). Recently, 'selfie' has been named word of the year 2013 by Oxford Dictionaries, a choice that reflects both a marked increase in frequency of

\footnotetext{
* To whom correspondence should be addressed. E-mail: m.bertamini@liverpool.ac.uk 
use and a change in prominence and register. Here we used selfies to test three known principles of composition for portraits.

Our starting point is that selfies provide a useful test bed for studying art production, perception, and naïve aesthetics. A recent paper used in-laboratory selfies by non-professional photographers to study posing preferences (Bruno and Bertamini, 2013), an issue usually addressed by observational studies of professional's works. As a (pseudo-artistic) real-life behavior, selfie-taking provides a way to test principles of art production in populations uncontaminated by academia, and in-lab selfie-taking represents an obvious way to standardize conditions and procedures. Our results using selfies taken in controlled conditions provide interesting information on the status of these principles, as well as on the psychological process of taking images of oneself.

The rationale for our study is straightforward. If principles of composition are rooted in psychological mechanisms governing visually perceived balance, non-professional photographers should tend to follow them. In other words, certain compositions should be chosen over others, as if certain spatial arrangements acted as 'attractors' for features of the photographed faces. This attracting effect should be visible, over individual differences and random error, in the distributions of feature positions. Importantly, moreover, this effect should be visible in individuals who never received formal training in photographic composition. We tested this prediction for three principles that have been cited and discussed in photography manuals and in the perception literature: the rule of thirds, the golden ratio rule, and the eye centering principle.

\subsection{Rule of Thirds}

The rule of thirds (ROT) was first mentioned and applied to landscapes by Smith (1797, p. 15-17). In photography handbooks, the ROT is widely reported as an important guideline for achieving overall balance in the picture and for highlighting picture elements (e.g., Lenman, 2005; Peterson, 2003). The ROT prescribes that the picture be divided into nine equally sized parts, by mentally tracing vertical and horizontal lines at the one-third and two-thirds of each side. Important compositional elements should then be placed along these lines (see Note 1). In the section on 'how to take the perfect selfie' of a recent web article (BBC News Magazine, 2013), a professional photographer summarized this suggestion in the following way: "try to think how to compose in thirds, like eyes a third of the way up, head a third of the way into the picture'. Although it has not been prominent in contemporary scientific analyses of aesthetics, the ROT is often employed in algorithms for assessments of image quality in computer vision algorithms (see e.g., Datta et al., 2006; Liu et al., 2010; Luo and Tang, 2008; Machajdik and Hanbury, 2010; Santella et al., 2006). 


\subsection{Golden Ratio Rule}

Two quantities $a$ and $b$ are said to form a golden ratio if their ratio is the same as the ratio of their sum to the larger of the two, that is, when $(a+b) / \operatorname{MAX}(a, b)$ is equal to $\operatorname{MAX}(a, b) / \operatorname{MIN}(a, b)$. The value of the golden ratio is 1.618 (with three decimals precision) therefore dividing an extent at approximately the $62 \%$ and the $38 \%$ point. The golden ratio has interesting mathematical properties, describes biological phenomena such as plant growth, and was used in Greek sculpture and architecture. Classical treatises of geometry (see e.g., Pacioli, 1509) as well as early studies of experimental aesthetics (Fechner, 1876) suggested that a golden ratio rule (GRR) might be used to achieve perceived balance. Consequently, and in contrast to the ROT, the GRR has been widely studied in academic circles. However, empirical studies have yielded mixed results (see e.g., Bertamini et al., 2011; Green, 1995; Höge, 1997; McManus and Weatherby, 1997; Ohta, 1999; Phillips et al., 2010; Plug, 1980). In addition, McManus and Weatherby (1997) reported wide inter-individual variability. When applied to a picture, the GRR states that the horizontal and vertical sides should be divided by lines placed either at about $62 \%$ or at $38 \%$ of the total extent (depending on where one wants the larger extent to be). This provides an alternative candidate rule for achieving aesthetically pleasing compositions: focal elements should be aligned with lines that divide the image according to the GRR. When applied to selfies, one would predict that eyes, or other notable features of a face, fall on one of these lines.

\subsection{Eye Centering Principle}

Tyler (1998; see also Tyler, 2007a) examined a corpus of portraits painted throughout the past 600 years and found evidence for an eye centering principle (ECP). According to the ECP, one of the eyes of the subject should be centered horizontally in the image. There is currently no consensus on the status of the ECP as a principle underlying the composition of portraits (McManus and Thomas, 2007; Tyler, 2007b), and the ECP has been generally ignored as a rule of composition in non-academic circles and professional photography. Nevertheless, the ECP provides a third alternative that might be applied to selfies. Selfie-takers might try to place one eye on the line that splits in half the image horizontally.

\subsection{Relationship Between the Three Rules}

It may be noted that the one-third and two-thirds of an extent (the ROT predictions) are rather close to its $38 \%$ and $62 \%$ (the GRR predictions). Moreover, applying the ECP to one eye could result in the other eye to also fall at $38 \%$ or $62 \%$ of the extent or thereabouts, especially if there is an additional preference for three-quarter poses (McManus and Humphrey, 1973) which might 
promote asymmetric placement of the face in the image. Thus, it is possible that the ROT and ECP are in fact simply user-friendly heuristics for applying the GRR. Perhaps a biologically ingrained association of the golden ratio with perceived balance is in fact the basis for all three rules of composition. This potential relationship between the three rules makes it even more interesting to test all three within the same set.

\section{Methods}

We tested if the ROT, the GRR, or the ECP account for compositional choices in a natural, widespread form of photographic production, the smartphone selfportrait ('selfie'). To this aim, we exploited a dataset of 388 selfies produced by students, colleagues, and acquaintances using the Apple iPhone (for details on the procedure for obtaining the self-portraits and other features of these images, see Bruno and Bertamini, 2013).

This dataset is especially useful as a means of investigating the psychological status of these rules. First, selfies taken by photographers who never received explicit training in photographic composition are a natural way to test whether compositional rules stem from spontaneous perceptual preferences. Second, selfie-taking is easy. Even without any training in photography, almost anyone can learn how to do it in a few minutes, and the availability of a preview screen and a front camera makes searching for pleasing compositions straightforward. Thus, although we selected individuals untrained in photography, our selfies were unlikely to be affected by lack of training in photography. Third, because the images consisted of self-portraits on mostly homogeneous backgrounds, the choice of potential relevant features in our selfies reduced to the main features of the face, like the eyes, the nose, and the mouth of the subject. Hence we did not need to rely on other methods, such as computing a saliency map (see Amirshahi et al., 2012), and their specific assumptions to define the main features of our stimuli. Note that evaluation of special locations within an image always suffers from the difficulty of arbitrary selection procedures. In this respect portraits have an advantage as the subject matter as the salient features of a face are relatively uncontroversial. Fourth, and final, our sample of self-portraits was collected to test completely different research questions (Bruno and Bertamini, 2013; Gionni, 2013) and is therefore free from potential selection biases by experimenters or demand characteristics that may affect participants when producing the images.

\subsection{Participants}

We collected 388 selfies from members of the University of Parma community and other acquaintances (167 males). All gave written informed consent to the use of their self-portrait for research (see Bruno and Bertamini, 2013, 
for Ethics Committee information). None of the participants was told that the study assessed compositional rules. In fact, most of them were collected for the purposes of two earlier studies, on posing preferences in self-portraiture (Bruno and Bertamini, 2013) and on the ergonomics of the preview screen on the iPhone (Gionni, 2013).

\subsection{Design}

Participants took their selfie in three conditions. In the 'portrait' condition (106 participants), they held the smartphone with the longer side in the vertical orientation; in the 'landscape' condition (202 participants), they held it with the longer side in the horizontal orientation. These two conditions were compared to test for effects of the picture frame on composition. In both, participants were left free to choose whatever pose and composition they liked. In the 'portrait forcing 3/4' condition, conversely, participants held the phone as in the portrait condition but were instructed to choose a three-quarter pose and to avoid full frontal images (80 participants). This third condition was included to test for effects of instructions on composition.

\subsection{Choice of Face Features for Analysis}

We analyzed the position of the following candidate face features that selfietakers might try to focus on while composing the picture: the right eye pupil and the left eye pupil; the nose center, as determined by locating the midpoint between the nostrils at the base of the nose; the right and the left mouth corners. To validate the choice of these features we also collected data using a questionnaire from a separate group of participants. In this paragraph we describe this small study. Forty participants were asked the following question: "With respect to a portrait, please rate how important are the following features of the face. Please use a scale from 1 (unimportant) to 10 (extremely important)". The features were: eyes, ears, nose, hair, chin, mouth. This sequence was presented in random order followed by a final item 'other please specify'. Only nine participants suggested another feature. The mean rating for each of the features was: eyes 8.97, mouth 7.56, nose 7.38, hair 7.17, ears 4.36, chin 5.36. We take these results as supporting the importance of eyes in people's intuitive reasoning about composition, followed by mouth and nose. Although people rated hair as important as well, this is a less localized feature, and variability in this feature is great (including the case of hair being absent). Therefore we focused our analysis on the position of eyes, mouth and nose.

\subsection{Analysis}

All images were inspected individually by the second and the third authors. For each image, we recorded the $x$ and $y$ coordinates of each candidate feature, scaled them to the total width or height of the frame, and converted to 
percentages. In addition, all coordinates were mirror-inverted such that they corresponded to the actual image that participants saw on the iPhone preview when composing the selfie (see Note 2). The analysis was then performed in three steps. First, we plotted the $x, y$ positions of each recorded feature separately for each condition and inspected the plots for obvious trends. Second, we compared the distributions of potentially relevant feature positions against the ROT, GRR, and ECP predictions by $t$-tests. These test assumed that all participants used one and the same focal feature, say, one of the eyes, and tried to place it on the imaginary line predicted by one of the rules. Thus, for instance, they might have tried to align the left eye with the two-thirds of the vertical side, the one-third of the horizontal, or the two-thirds of the horizontal (ROT); to the $62 \%$ of the vertical side, to the $62 \%$ of the horizontal, to the $38 \%$ of the horizontal (GRR); they might have tried to center it horizontally (ECP). Third, we entertained the more complex hypothesis that participants differ in their choice of features to place on 'focal' positions according to a given compositional rule. In this more complex hypothesis, the data resulted from a mixture of different behaviors, for instance, participants may follow the rule "place the most important feature on a thirds intersection", but some might choose one eye as the most important feature, others might choose the nose, and so on. To test this hypothesis, we computed the number of images whereby at least one of the potential features fall on one of the potential focal positions, repeating the computation for different 'tolerance areas' around the focal points.

\section{Results and Discussion}

The results are summarized in Figs 1, 2, and 3 for the portrait, landscape, and portrait forcing $3 / 4$ conditions. A quick perusal of the three diagrams suggest systematic deviations from the ROT and the ECP predictions. The datapoints for all five features do not show clustering along one of the corresponding lines or, for the eyes and the mouth corners, bimodalities that would arise if different subsets of participants tried to follow the rule with opposite (left or right) features. The difference between the distributions and the predicted lines is graphically clear even without statistical testing. Thus, based on inspection of the diagrams in the figures, we rule out that all participants choose one (and the same) feature and attempted to align it along one of the predictions of the ROT or of the ECP. When compared to the predictions of the GRR, conversely, the data from the three conditions show patterns that require testing. In addition, all three diagrams seem to show a consistent centering of the nose. We examine these two trends in the following paragraphs.

In the portrait condition (Fig. 1), averages for the distributions of three features appear to align with the GRR predictions: the right eye $($ mean $=0.389)$ and the right mouth corner (mean $=0.396$ ) horizontally, plus the left mouth 


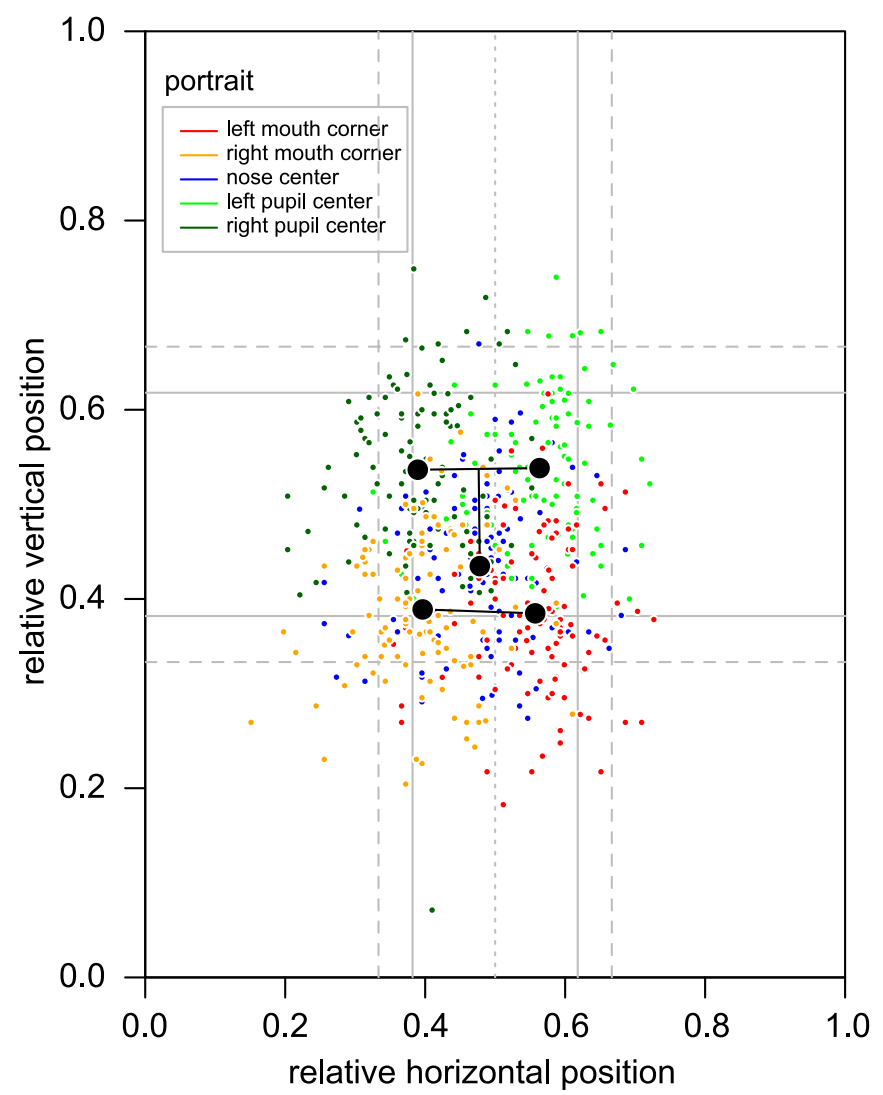

Figure 1. Portrait condition results. Relative positions of five candidate face features (right eye pupil, left eye pupil, nose center, right mouth corner, left mouth corner) are plotted against the predictions of the ROT (dashed grey lines), GRR (solid grey), and ECP (dotted grey). Right and left refer to the self-portrait facing towards the viewer, not to the position relative to the page. Thus the right eye is actually plotted on the left in the diagram. This figure is published in color in the online version.

corner $($ mean $=0.385)$ and the right mouth corner $($ mean $=0.389)$ vertically. Tests of the null hypothesis that the horizontal and vertical means $=0.382$ confirmed that the means of these distributions are not statistically different from the GRR predictions, $t(105)=1.05,1.9,0.97$, and $0.35, p=0.29,0.06,0.39$, and 0.73 for each in the above order. In the landscape condition (Fig. 2), however, the right eye and right mouth corner averages do not align vertically with the GRR prediction, $t(201)=7.7$ and 9.5 , both $p$ 's $<0.00001$, whereas only the right and left mouth corners continue to align horizontally to the GRR, both $t(201)<1$ for both. In the landscape forcing 3/4 condition (Fig. 3), finally, no average appears to align even with the GRR prediction. The closest to the first horizontal GRR line is the right eye. However, even this mean is 


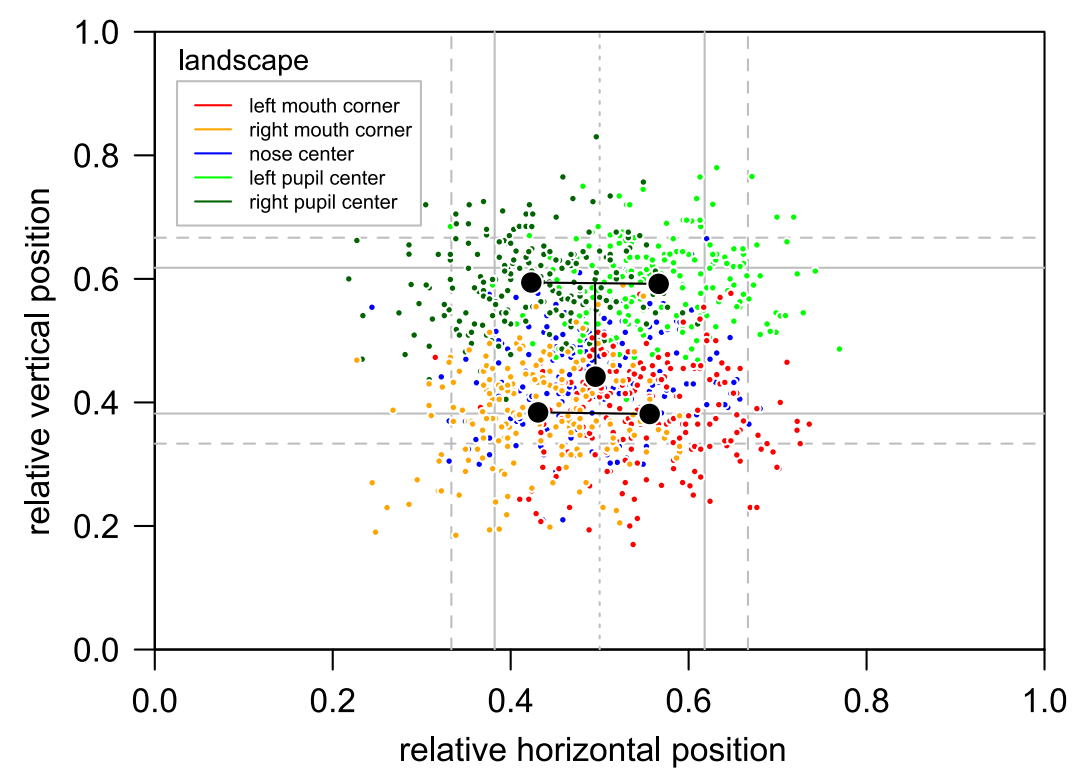

Figure 2. Landscape condition results. All plotting conventions as in Fig. 1. This figure is published in color in the online version.

statistically different from $0.382, t(79)=4.3, p<0.00005$. Thus, there was some evidence that participants aligned the mouth relative to the lower vertical GRR line in two of the conditions, and the right eye and mouth corner to the left horizontal GRR line in one of the conditions. However, neither of these trends survived a simple manipulation of instructions as in the portrait forcing 3/4 condition. Thus, the evidence for the GRR is weak, and may have resulted from averaging artifacts as the portrait and landscape conditions included some frontal selfies, whereas the portrait forcing 3/4 did not.

Although not predicted by any of the rules we have considered, the most consistent trend in the data seems to be a tendency to center the nose horizontally. The $x$ coordinates of the nose were $0.478,0.495$, and 0.496 for portrait, landscape, and portrait forcing $3 / 4$, respectively. After testing, however, the first of these three means proved statistically different from 0.5 , $t(105)=-2.7, p<0.0081$. Only the second and third were statistically consistent with centering, $t(201)$ both $<1$. Statistical significance notwithstanding, it is especially interesting that the nose, on the average, tended to be centered horizontally. Presumably, this trend arises from the fact that selfies in the three conditions were mixtures of different poses, including left- or rightsided 3/4 poses (portrait condition forcing 3/4) plus frontal and slightly rotated poses (portrait and landscape). These mixtures, however, showed a small (but statistically reliable) bias for showing more the right preview-image cheek 


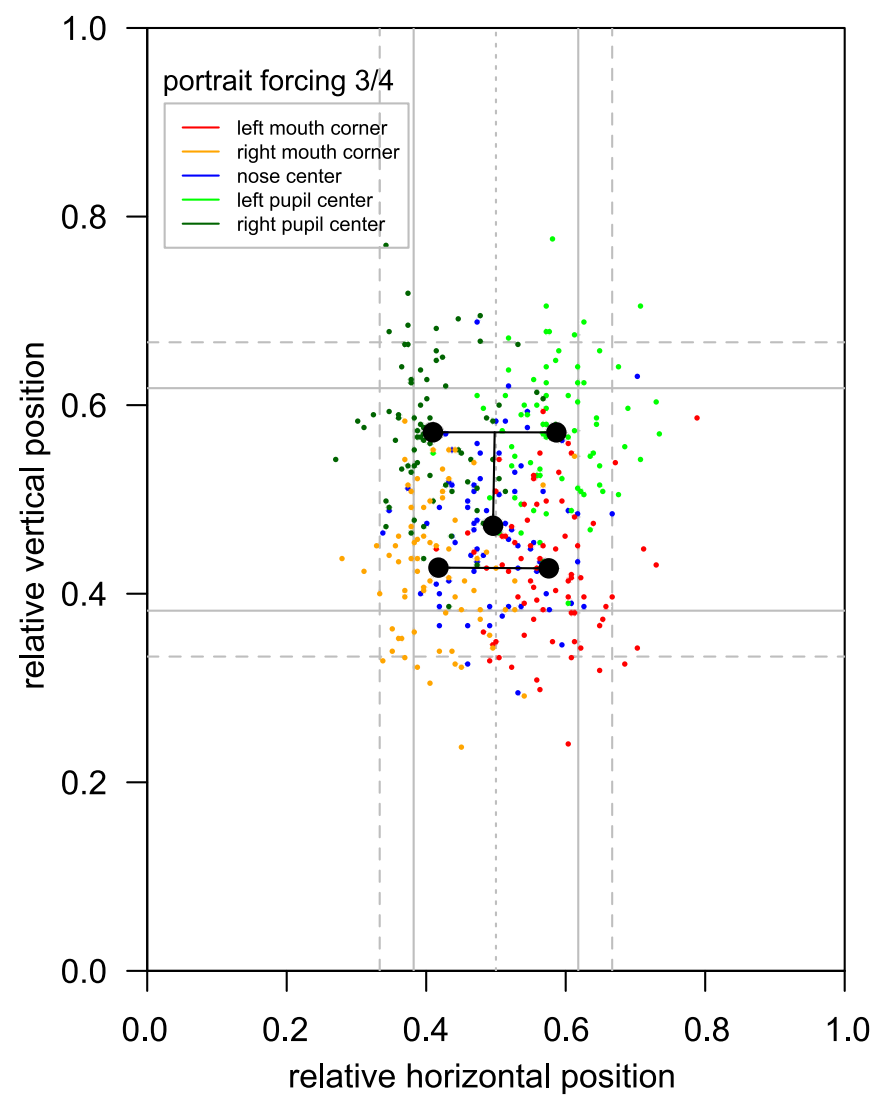

Figure 3. Portrait forcing 3/4 condition results. All plotting conventions as in Figs 1 and 2. This figure is published in color in the online version.

(Bruno and Bertamini, 2013). This mix might yield an average position of the nose around the horizontal center, and an asymmetry in the position of the other features related to the right side bias. This is essentially what is seen in the diagrams, most clearly for the portrait condition and to a lesser extent for the other two.

Thus, statistical tests on the central tendency of the distributions of each feature position in the image failed to reveal consistent tendencies to place them on the lines predicted by the ROT, the ECP, and the GRR. These tests, however, assume that all or at least most of the participants choose to focus on the same feature and attempted to place it on one of the predicted lines. This possibility is supported by the common intuition that eyes are the most salient feature of a human face, and by the ranking of feature importance that we observed in our preliminary survey. However, the assumption may not be correct. Different participants may have chosen to focus on different features, 
such that some attempted to align the eyes on a given predicted line, others to align the mouth, and so on. Under this more complex scenario, the data result from a mixture of behaviors that would not be detected by tests on the distribution of each single feature.

There is, however, an alternative approach that proves useful to evaluate whether our selfies support the more complex scenario. After defining a tolerance area, we can count how many selfies had at least one of the relevant features within this area around one of the predicted lines. We might assume, for instance, that a reasonable tolerance is around $2 \%$ of the total width or height of the image. We would then count as a 'hit' in favor of the ROT all images having at least one feature falling on one of the four strips centered on the ROT predictions, and having width and height equal to $2 \%$ of the total image width or height. From the count we can then compute the percentage of images that are consistent with a given prediction. By repeating this process for different tolerance values, we can also evaluate how large the tolerance would need to be, before the, say, $50 \%$, or $80 \%$ of all images would be taken to be consistent with a compositional rule. Although comparison with a chance model is difficult, especially given the relatively unconstrained instructions, these figures provide us with empirical estimates of the spatial precision of the predictions associated with each compositional rule.

The results of the above-described analysis are presented in Fig. 4 for the ROT and GRR rules. We did not perform a similar analysis for the ECP as the ECP makes predictions about eye placement only. The graphs again provide little support for either the ROT or the GRR. With a $2 \%$ tolerance, a sizable
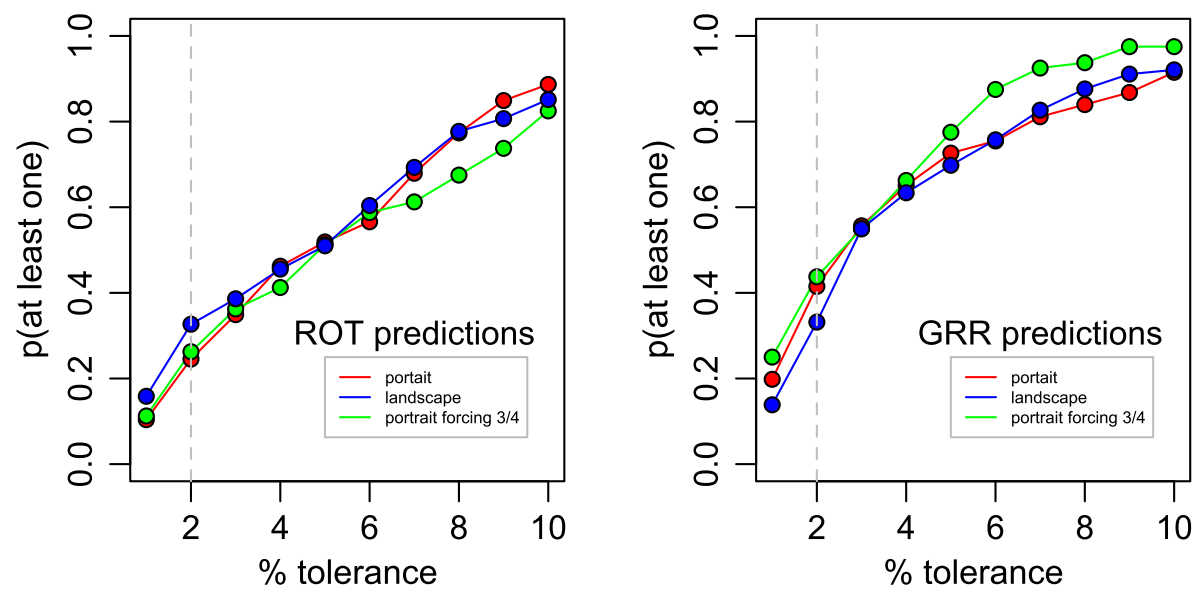

Figure 4. Relative frequencies of selfies with at least one feature falling within a given tolerance area around the lines predicted by the ROT (left) and the GRR (right). With a $2 \%$ tolerance, about $30 \%$ of images were consistent with the ROT predictions; about $40 \%$ were consistent with the GRR predictions. This figure is published in color in the online version. 
strip around predicted lines, only about $30 \%$ and $40 \%$ of all images can be taken as supporting the ROT or the GRR, respectively. Focussing on the ROT predictions, the tolerance must be around $8 \%$ before $80 \%$ of images can be considered hits. The same criterion yields a tolerance of about $6 \%$ for the GRR predictions.

\section{Conclusions}

Rules of composition for the visual arts, such as the ROT and the GRR, are often discussed and prescribed in non-academic circles, for example in relation to professional or non-professional photography. Alternative possibilities, such as the ECP, have been investigated in empirical studies, as has the GRR. However, understanding of the status of these rules remains limited. In this paper, we used a database of selfies, smartphone self-portraits taken in controlled conditions by non-professionals, to test whether the placement of the main facial features is consistent with known principles of photographic composition. If these rules were rooted in the psychology of aesthetics experiences, one would expect to see a tendency to follow them even in participants lacking training in photographic composition.

Our results do not provide evidence supporting this expectation. In absolute terms, our results revealed systematic deviations from predictions based on all the rules we examined, both under the more restrictive assumption that all participants focus on one and the same feature, and under the broader assumption that different participants focus on different features. In relative terms, the GRR seems to make somewhat better predictions than the ROT or the ECP. Even for this rule, however, the evidence is weak at best. It may suggest an approximate tendency of face features to be within a relatively large area around the GRR predicted lines. This tendency may reflect a somewhat imprecise, broad principle of visual balance, which we might perhaps dub a 'fuzzy golden ratio rule'. More plausibly, it may reflect a general bias to position the face more or less in the center of the picture, causing at least one feature to fall in the vicinity of the central portions of the GRR lines.

Although these observations provide interesting data on selfie-taking, as well as on the status of compositional rules, our conclusions have limitations. First, they may apply only to self-portraits, and not to other photographic genres. This leads to an obvious additional empirical question, that could be answered by collecting other types of photographs from non-professional photographers in controlled conditions. For instance, it may be that a stronger tendency to use golden ratios is visible in landscape photographs. Second, there may be more complex ways that compositional rules could be applied to distributed features in a picture. For instance, golden ratios can be formed between the long and short sides of rectangles as opposed to two collinear 
parts of a whole segment. It is sometimes argued that the use of such 'golden rectangles' is apparent in the relative sizes, rather than placement, of elements in paintings such as those by Leonardo da Vinci or Piet Mondrian. Although it is not obvious how this idea might be applied to the composition of selfies, it might be of interest to explore this possibility. Finally, there may be other, more general compositional principles that are related to perceptual mechanisms. For instance, it is often noted that asymmetric compositions possess greater perceptual dynamics. It may be that rules such as the ROT, GGR, or ECP are merely to discourage dividing the subject exactly in half vertically or horizontally, which is generally considered a symptom of poor composition (Krages, 2005). Interestingly, our participants tended to place the face around the centre of the frame, but most of them avoided frontal poses in favour of $3 / 4$ or at least slightly rotated head positions (see Bruno and Bertamini, 2013).

In conclusion, these findings argue against the hypothesis that the ROT, GRR, or ECP stem from fundamental principles of visual perception. These rules of composition do not seem to come natural to non-professional photographers, and may therefore be learned and, to some extent, conventional. Although their function remains unclear, we suggest that the ROT, GRR, and ECP should be regarded merely as practical heuristics for counterbalancing the tendency to place the main subject in the very centre of the frame.

\section{Acknowledgements}

This study was supported in part by the ESRC (MB) and a FIL2012 small grant from the University of Parma (NB).

\section{Notes}

1. Suggesting that the ROT is widely regarded as useful, most modern cameras include options to superimpose thirds-lines on a preview screen. However, some ambiguity remains as to what exactly is the rule. According to the Oxford Companion to the Photograph, 'focal points' of a picture should be placed on the thirds intersections, not just on the lines (Lenman, 2005). This interpretation is more restrictive, but here we will test the more general hypothesis that important features are placed along lines. Refuting this more general hypothesis implies that the restricted one is also wrong.

2. When taking a selfie with a smartphone front-camera, the preview screen shows your image as if you were looking at yourself in a mirror. When the image is saved, conversely, it is saved as if the picture had been taken by the back-camera from the viewpoint of another photographer. Thus, the saved image does not in fact correspond to the choice one makes when composing the selfie, but to its mirror reversal. Remarkably, many users 
never notice this aspect of the functionality of their smartphone. We have not been able to find information on the reasons for this design, which may or may not aid in 'taking a perfect selfie'. This may be an interesting empirical question for cognitive ergonomists.

\section{References}

Amirshahi, S. A., Redies, C. and Denzler, J. (2012). Assessment of the Rule of Thirds in Photographs Taken by Amateurs. Paper presented at VSAC Conference, Alghero, Italy.

BBC News magazine (2013). Self-portraits and social media: the rise of the 'selfie'. 7 June 2013. http://www.bbc.co.uk/news/magazine-22511650.

Bertamini, M., Bennett, K. M. and Bode, C. (2011). The anterior bias in visual art: the case of images of animals, Laterality 16, 673-689.

Bruno, N. and Bertamini, M. (2013). Self-portraits: smartphones reveal a side bias in non-artists, PLoS One 8, e55141.

Datta, R., Joshi, D., Li, J. and Wang, J. Z. (2006). Studying aesthetics in photographic images using a computational approach, in: ECCV 2006 Lecture Notes in Computer Science, A. Leonardis, H. Bischof and A. Pinz (Eds), pp. 288-301. Springer Verlag, Berlin, Heidelberg, Germany.

Fausing, B. (2013). Selfies and the Search for Recognition. See for Your Selfie. Web article retrieved on November 1, 2013, http://www.academia.edu/4418191/Selfies_and_the_Search_ for_Recognition._See_for_your_Selfie.

Fechner, G. Th. (1876). Vorschule der Aesthetik, 2nd edn. Breitkopf and Härtel, Leipzig, Germany.

Gionni, R. (2013). Autoritratti con lo smartphone: una Ricerca Empirica. Unpublished undergraduate thesis, Università di Parma, Italy.

Green, C. D. (1995). All that glitters: a review of psychological research on the aesthetics of the golden section, Perception 24, 937-968.

Höge, H. (1997). The golden section hypothesis: its last funeral, Empir. Stud. Arts 15, 233-255.

Houghton, D., Joinson, A., Caldwell, N. and Marder, B. (2013). Tagger's Delight? Disclosure and Liking in Facebook: the Effects of Sharing Photographs amongst Multiple Known Social Circles, Discussion Paper, University of Birmingham Discussion Paper Series, Birmingham.

Krages, B. (2005). Photography: The Art of Composition. Allworth Press, New York, NY, USA.

Lenman, R. (Ed.) (2005). The Oxford Companion to the Photograph. Oxford University Press, Oxford, UK.

Liu, L., Chen, R., Wolf, L. and Cohen-Or, D. (2010). Optimizing photo composition, Comput. Graph. Forum 29, 469-478.

Luo, Y. and Tang, X. (2008). Photo and video quality evaluation: focusing on the subject, in: ECCV 2008, Part III, LNCS 5304, D. Forsyth, P. Torr and A. Zimmerman (Eds), pp. 386399. Springer Verlag, Berlin, Heidelberg, Germany.

Machajdik, J. and Hanbury, A. (2010). Affective image classification using features inspired by psychology and art theor, Paper presented at the International Conference on Multimedia, New York, NY, USA.

McManus, I. C. and Humphrey, N. K. (1973). Turning the left cheek, Nature 243, 271-272. 
McManus, I., Stöver, K. and Kim, D. (2011). Arnheim's Gestalt theory of visual balance: examining the compositional structure of art photographs and abstract images, $i$-Perception 2 , 615-647.

McManus, I. C. and Thomas, P. (2007). Eye centring in portraits: a theoretical and empirical evaluation, Perception 36, 167-182; discussion 183-188.

McManus, I. C. and Weatherby, P. (1997). The golden section and the aesthetics of form and composition: a cognitive model, Empir. Stud. Arts 15, 209-232.

Ohta, H. (1999). Preferences in quadrangles reconsidered, Perception 28, 505-517.

Pacioli, L. (1509). De divina proportione (English: On the Divine Proportion), Luca Paganinem de Paganinus de Brescia (Antonio Capella): Venice.

Peterson, B. (2003). Learning to See Creatively: Design, Color and Composition in Photography. Amphoto Press, New York, NY, USA.

Phillips, F., Norman, J. F. and Beers, A. M. (2010). Fechner's aesthetics revisited, Seeing Perceiving 23, 263-271.

Plug, C. (1980). The golden section hypothesis, Am. J. Psychol. 93, 467-487.

Russell, P. A. (2000). The aesthetics of rectangle proportion: effects of judgment scale and context, Am. J. Psychol. 113, 27-42.

Santella, A., Agrawala, M., deCarlo, D., Salesin, D. and Cohen, M. (2006). Gaze-based interaction for semi-automatic photo cropping, Paper presented at the SIGCHI Conference on Human Factors in Computing Systems, New York, NY, USA.

Smith, J. T. (1797). Remarks on Rural Scenery, with Twenty Etchings of Cottages, from Nature, and Some Observations and Precepts Relative to the Picturesque, printed for, and sold by Nathaniel Smith, Ancient Print Seller, at Rembrandt's Head, May's Buildings, St. Martin's Lane, and I. T. Smith, at No 40 Trith Street, Soho, London, UK.

Tyler, C. W. (1998). Painters centre one eye in portraits, Nature 392, 877-878.

Tyler, C. W. (2007a). Some principles of spatial organization in art, Spat. Vis. 20, 509-530.

Tyler, C. W. (2007b). Eye-centering in portraits: reply to McManus and Thomas, Perception 36, 183-188. 